\title{
O uso da tomografia computadorizada de baixa dose (TCBD) no rastreio de câncer de pulmão: revisão narrativa
}

\author{
The use of low dose computed tomography (LDCT) in the screening of lung cancer: \\ narrative review
}

El uso de tomografía computarizada de baja dosis (TCBD) en la detección del cáncer de pulmón: revisión narrativa

Laerte de Paiva Viana Filho1, João Pedro Costa Apolinário', Daniela Teixeira Ribeiro², Gabriele Maria Braga ${ }^{2}$, Laura de Araujo Soares ${ }^{3}$, Pedro Paulo Teixeira Baraky ${ }^{4}$, Yllana Ferreira Alves da Silva ${ }^{5}$.

\section{RESUMO}

Objetivo: Avaliar o uso da tomografia computadorizada de baixa dose (TCBD) no rastreamento do câncer de pulmão (CP). Revisão bibliográfica: O CP representa a neoplasia com maior incidência e mortalidade no mundo e caracteriza-se por rápida progressão e diagnóstico tardio, fatores que limitam as opções terapêuticas e contribuem para a baixa sobrevida dos pacientes. Medidas de rastreio mostram-se favoráveis em pacientes assintomáticos com alto risco de neoplasia, contribuindo com um prognóstico favorável. A TCBD revela-se como a principal ferramenta de rastreio, apresentando maior sensibilidade na detecção precoce de neoplasia de pulmão e de câncer em estágio I, quando comparada à triagem por radiografia torácica. Entretanto, fatores como a relação custo-benefício, a alta exposição à radiação ionizante e risco de falsos positivos representam empecilhos que devem ser analisados e considerados antes da implementação da triagem generalizada por TCBD. Considerações finais: A TCBD é objeto de estudo devido à grande relevância de seu uso na triagem de câncer de pulmão, decorrente de análises bibliográficas e pesquisas anteriores.

Palavras-chave: Neoplasias pulmonares, Programas de rastreamento, Tomografia.

\section{ABSTRACT}

Objective: To evaluate the use of low-dose computed tomography (TCBD) in the screening of lung cancer (CP). Bibliographic review: PC represents the neoplasm with the highest incidence and mortality in the world and is characterized by rapid progression and late diagnosis, factors that limit therapeutic options and contribute to low patient survival. Screening measures are favorable in asymptomatic patients at high risk of cancer, contributing to a favorable prognosis. TCBD proves to be the main screening tool, presenting greater sensitivity in the early detection of lung cancer and stage I cancer, when compared to screening by chest radiography. However, factors such as the cost-benefit ratio, high exposure to ionizing radiation and risk of false positives represent obstacles that must be analyzed and considered before the implementation of generalized screening by TCBD. Final considerations: TCBD is the object of study due to the great relevance of its use in screening for lung cancer, resulting from bibliographic analyzes and previous research.

Keywords: Lung neoplasms, Mass screening, Tomography.

\footnotetext{
${ }^{1}$ Pontifícia Universidade Católica de Minas Gerais (PUC-MG), Betim - MG. *E-mail: laerteviana07@gmail.com ${ }^{2}$ Centro Universitário de Belo Horizonte (UniBH), Belo Horizonte - MG.

${ }^{3}$ Faculdade Ciências Médicas de Minas Gerais (CMMG), Belo Horizonte - MG.

${ }^{4}$ Universidade Federal dos Vales do Jequitinhonha e Mucuri (UFVJM), Teófilo Otoni - MG.

${ }^{5}$ Centro Universitário Uninovafapi (UNINOVAFAPI), Teresina - PI.
} 


\section{RESUMEN}

Objetivo: Evaluar el uso de tomografía computarizada de baja dosis (TCBD) en la detección del cáncer de pulmón (CP). Revisión bibliográfica: PC representa la neoplasia con la mayor incidencia y mortalidad en el mundo y se caracteriza por una rápida progresión y diagnóstico tardío, factores que limitan las opciones terapéuticas y contribuyen a la baja supervivencia del paciente. Las medidas de detección son favorables en pacientes asintomáticos con alto riesgo de cáncer, lo que contribuye a un pronóstico favorable. TCBD demuestra ser la principal herramienta de detección, presentando una mayor sensibilidad en la detección temprana de cáncer de pulmón y cáncer en estadio I, en comparación con la detección por radiografía de tórax. Sin embargo, factores como la relación costo-beneficio, la alta exposición a la radiación ionizante y el riesgo de falsos positivos representan obstáculos que deben analizarse y considerarse antes de la implementación de la detección generalizada por TCBD. Consideraciones finales: TCBD es el objeto de estudio debido a la gran relevancia de su uso en la detección del cáncer de pulmón, como resultado de análisis bibliográficos e investigaciones previas.

Palabras clave: Neoplasias pulmonares, Tamizaje masivo, Tomografia.

\section{INTRODUÇÃO}

O Câncer de Pulmão (CP) é a neoplasia com maior incidência e mortalidade no mundo. Responsável por $11,6 \%$ dos casos e por $18,4 \%$ de todas as mortes de causa neoplásica, a doença tem como um dos principais fatores de risco a exposição crônica ao tabaco. Além disso, devido a apresentação clínica heterogênea e frequentemente ausente nos estágios iniciais, O CP caracteriza-se por rápida progressão, principalmente devido ao diagnóstico tardio, muitas vezes em estágio avançado, o que limita as opções terapêuticas e contribui para um prognóstico com baixa sobrevida dos pacientes (NHS, 2019; LIU Y, et al., 2019; TANG X, et al., 2019).

As células neoplásicas do CP decorrem de alterações nos mecanismos reguladores que controlam a proliferação e a homeostase normal das células, resultado de uma série de alterações determinadas geneticamente. Após o desenvolvimento do câncer primário, o acúmulo contínuo de anormalidades genéticas e epigenéticas adquiridas durante a expansão clonal influencia os processos de invasão, metástase e resistência à terapêutica (HASSAN LA, et al., 2015).

A clínica do CP é diversa, e na maioria das vezes, assintomática até estágios avançados. O surgimento de tosse ou alteração em padrão pré-existente, é a manifestação clínica mais prevalente. A pneumonia de repetição em mesmo local anatômico ou exacerbações frequentes da doença pulmonar obstrutiva crônica (DPOC) também sugerem neoplasia. Dispneia, hemoptise, angina e rouquidão podem estar presentes. Como manifestações secundárias a metástases extratorácicas, pacientes podem cursar com perda de peso, anorexia e fadiga (NASIM F, et al., 2019).

Em relação a sua classificação, ela é feita em dois grupos: o Carcinoma Pulmonar de Células Pequenas (CPCP) e o Carcinoma de Pulmão de Células Não Pequenas (CPCNP). O segundo grupo, caracterizado pelo crescimento desordenado das células epiteliais, representa $85 \%$ dos casos diagnosticados de CP e inclui o adenocarcinoma, o carcinoma de células escamosas e o carcinoma de células grandes, sendo o adenocarcinoma a manifestação mais comum da neoplasia pulmonar correspondendo a $38 \%$ dos novos diagnósticos (ORTEGA-GÓMEZ A, et al., 2016; INCA, 2018; SILVA HAP, et al., 2019; WOODARD G, et. al., 2016).

O tratamento depende do estágio do $\mathrm{CP}$, de modo que pacientes em estágio I ou II são usualmente tratados com ressecção cirúrgica completa. Quando a abordagem cirúrgica não está indicada, considera-se a utilização da radioterapia. Verificou-se que procedimentos de ablação térmica percutânea, como crioablação, ablação por radiofrequência e por micro-ondas, são opções úteis de tratamento no cenário da terapia de resgate pós cirurgia, radioterapia ou quimioterapia ou para cuidados paliativos em CP avançado. Já para os pacientes de CPCP que não podem ser submetidos a cirurgia, a quimioradioterapia mostra-se como a primeira opção de tratamento (DUMA N, et al., 2019; HONGLIN Z, et al., 2018). 
Segundo dados mais recentes do Instituto Nacional de Câncer, o câncer de pulmão foi responsável por 27.929 mortes no ano de 2017, sendo a taxa de sobrevida relativa em cinco anos de apenas $18 \%$. No entanto, quando detectado em estágios iniciais (estágio I/II), situação observada em apenas $16 \%$ dos casos, a taxa de sobrevida de cinco anos do câncer de pulmão eleva-se para $56 \%$.

Diante desse cenário, medidas de rastreio mostram-se favoráveis em pacientes assintomáticos com alto risco de neoplasia, sobretudo os pacientes maiores de 55 anos e com níveis de tabagismo superior a 30 anosmaço, de acordo com o "National Lung Screening Trial" (NLST). Desse modo, a detecção precoce da doença pode gerar grandes impactos na redução da mortalidade e na melhora da sobrevida por câncer de pulmão (JAMNIK S, et al., 2012; LIU Y, et al., 2019).

Inicialmente, o instrumento de rastreio estudado era a radiografia de tórax com e sem citologia de escarro, que não mostrava, no entanto, benefícios significativos. Por sua vez, em 2011, o NLST sugeriu que a Tomografia Computadorizada de Baixa Dose (TCBD) estaria associada a uma diminuição de $20 \%$ na mortalidade por neoplasia pulmonar quando comparada à radiografia de tórax nos Estados Unidos. Contudo, ainda não há protocolos bem estabelecidos orientando o rastreamento de rotina para o CP (JAMNIK S, et al., 2012; WENDER R, et al., 2013).

Nesse sentido, este estudo se propõe a revisar a literatura existente sobre o assunto, avaliando os impactos, os benefícios e as limitações da adoção da TCBD como exame de rotina para o rastreamento de CP. Além disso, dispõe-se a estimular o desenvolvimento de mais ensaios clínicos sobre o tema no Brasil.

\section{REVISÃO BIBLIOGRÁFICA}

Dentre os novos casos de neoplasia no Brasil, a estimativa é que no ano de 2020, excluindo-se o câncer de pele não melanoma, o CP corresponderá a 9,1\% entre os homens e a 5,6\% entre as mulheres, totalizando 32.960 casos. Destaca-se essa neoplasia, ainda, como o segundo tipo de câncer, no Brasil, de maior incidência em ambos os sexos, excluindo-se o câncer de pele não melanoma. Dessa forma, configura-se uma questão de saúde de proporção e relevância global, sendo classificada, ainda, como uma das principais causas de morte evitáveis no mundo (INCA, 2020).

O tabagismo relaciona-se a cerca de $85 \%$ dos casos diagnosticados de CP e a aproximadamente $90 \%$ das mortes por tal etiologia, aumentando o risco relativo para desenvolvimento de carcinoma pulmonar em cerca de 25 vezes comparado ao risco em não fumantes. Os principais fatores de risco incluem a carga tabágica (maior que 30 anos/maço) e a idade do paciente. A exposição passiva à fumaça e a exposição ocupacional a agentes físico-químicos como asbesto e sílica também configuram fatores de risco ao $\mathrm{CP}$ (INCA, 2020).

Segundo dados do Surveillance, Epidemiology and End Results (SEER) do National Cancer Institute ( $\mathrm{NCl}$ ), entre os anos de 2010 a 2015, apenas $17 \%$ dos diagnósticos de CP foram feitos enquanto o tumor ainda estava localizado, enquanto $57 \%$ dos casos foram diagnosticados já com metástase distante.

Quando avaliada a sobrevida dos pacientes, aqueles diagnosticados com acometimento localizado tinham uma sobrevida em 3 anos aproximadamente 30\% superior àqueles pacientes diagnosticados já com metástases a distância. Diante desse cenário, o diagnóstico precoce do câncer tem o potencial de gerar grandes impactos na redução da mortalidade e na melhora da sobrevida por câncer de pulmão.

\section{História do rastreio}

Os estudos sobre o rastreio do CP iniciaram-se com a análise da radiografia de tórax como método de escolha, a princípio em Londres no ano de 1969, em um estudo com 55.000 indivíduos ao longo de 3 anos, seguido por outras pesquisas ao longo da década de 1970 nos Estados Unidos e República Tcheca.

Mais recentemente, o Prostate, Lung, Colorectal, and Ovarian (PLCO) também conduziu um estudo de rastreamento similar entre 1993 e 2001, contudo, foi consenso que o método não se mostrou viável para a redução da mortalidade por neoplasia de pulmão, visto que os resultados não demonstraram alteração estatisticamente significante (JAMNIK S, et al., 2012; OKEN MM, et al., 2011). 
Nos anos 1990, a introdução da tecnologia da Tomografia Computadorizada (TC), trouxe novas possibilidades para a triagem, e a partir de então estudos vêm buscando provar sua eficácia. O Early Lung Cancer Action Project (ELCAP) foi o primeiro a relatar a sensibilidade seis vezes maior atribuída à TCBD em relação a radiografia de tórax, com capacidade de detectar lesões parenquimatosas pulmonares menores que $1 \mathrm{~cm}$. Também foi verificado que a maioria dos tumores detectados pela TC tinham potencial de serem ressecáveis, visto que foram classificados em estágios precoces da doença ( $85 \%$ eram estádio I) (JAMNIK S, et al., 2012).

\section{Resultados dos trials}

Em busca de evidências científicas acerca do novo método, são conduzidos Ensaios Clínicos Randomizados (ECR) em diversos países, sendo o American National Lung Screening Trial (NLST) o primeiro a ser concluído. No Brasil, foi iniciado em 2013 o First Brazilian Lung Cancer Screening Trial (BRELT1), conduzido pelo Hospital Israelita Albert Einstein, um ECR que ainda está em andamento, porém já divulgou resultados iniciais (WENDER R, et al., 2013; SANTOS RS, et al., 2014, 2016).

Uma metanálise de 9 desses ECR ratifica tais achados ao demonstrar que a TCBD de fato teve uma vantagem absoluta em comparação com a radiografia torácica na triagem, tanto para encontrar uma proporção maior de neoplasia de pulmão quanto de câncer de estágio I, proporcionando uma redução da mortalidade específica por CP. No tocante às mortes por todas as causas, a revisão afirma não ter encontrado efeito protetor. A hipótese levantada é que os pacientes, em sua maioria tabagistas, estão sob risco de muitas doenças cardiovasculares, importantes causas de morte nos mais velhos (TANG X, et al., 2019).

A grande maioria dos estudos a respeito do rastreamento de câncer de pulmão por TCBD foi realizada em países onde a incidência de tuberculose, um dos principais exemplos de doença granulomatosa, é cerca de 5 a 10 vezes inferior a observada no Brasil e em países como Índia, China e Rússia.

Assim, foram levantadas questões a respeito da aplicabilidade e eficiência da técnica no rastreamento do CP em países onde a incidência de doenças granulomatosas era elevada, o que poderia implicar na possível detecção de numerosos nódulos benignos de causa inflamatória na população estudada, situação que pode predispor a intervenções cirúrgicas desnecessárias e aumento de resultados falsos-positivos ao rastreamento. Assim, como forma de comprovar a eficiência do método no contexto de tais países, foi finalizada no Brasil em 2016 a fase inicial do First Brazilian Lung Cancer Screening Trial (BRELT1) (SANTOS RS, et al., 2014, 2016).

O estudo demonstrou, em suma, que a prevalência de nódulos pulmonares rastreados foi muito superior à observada em outros estudos, incluindo o NLST. No entanto, o número de participantes que receberam indicação para realização de biópsia cirúrgica e que foram efetivamente diagnosticados com câncer de pulmão foi semelhante a outros relatos. Sugeriu-se, portanto, que a prevalência da doença granulomatosa não tenha elevado o número de resultados falso-positivos com alta suspeita de câncer de pulmão como o previsto, comprovando, assim, a também aplicabilidade e efetividade do método nessa população e, portanto, no Brasil (SANTOS RS, et al., 2014, 2016).

\section{Resultados por sexo}

A análise dos resultados também aponta para uma heterogeneidade quanto a redução de mortalidade entre os sexos desde os dados do NLST, que são ratificados pelos resultados preliminares do Dutch-Belgian Lung Cancer Screening trial ("NELSON"), no qual a triagem por TCBD indicou uma diminuição mais pronunciada da mortalidade entre mulheres do que entre homens ( $H R=0,94 ; \mathrm{IC} 95 \%$ : $0,54-1,61 ; p=0,81$; heterogeneidade $=0,09$ ).

Os relatórios preliminares do NELSON também sugerem uma redução mais acentuada da mortalidade entre o sexo feminino, a depender do tempo de seguimento, do que entre o masculino. De maneira similar, o German Lung cancer Screening Intervention ("LUSI") sugeriu uma redução da mortalidade unicamente entre as mulheres, descrevendo uma diferença significativa, contudo limítrofe (heterogeneidade $=0,09$ ). Uma possível explicação se relacionaria aos subtipos de tumores pulmonares, que são quantificados de maneira diferente em ambos os gêneros (BECKER N, et al., 2019). 


\section{Intervalo ideal de triagem}

A metanálise de Tang et al. (2019) lida com o problema da dissemelhança entre os ECRs, mas se propõe a avaliar qual seria o intervalo com ótimo potencial de triagem. No grupo de triagem anual com TCBD, encontraram-se mais cânceres de pulmão quando comparado tanto com o de triagem anual com raios- $X$ de tórax quanto com o grupo sem triagem, e a diferença foi estatisticamente significativa.

Em contrapartida, a alternativa de triagem bienal com TCBD não apresentou benefício relevante estatisticamente sobre o rastreio anual com raios- $X$ de tórax ou sobre o grupo sem triagem. A triagem anual por tomografia, portanto, é apontada como eficaz para encontrar mais neoplasias pulmonares em estágio inicial. Entretanto, esta conclusão carece de ser ratificada por mais estudos sobre os aspectos positivos e negativos desse intervalo de triagem.

Um intervalo diferente foi analisado pelo estudo NELSON: usando TCBD, a triagem com intervalo de 2,5 anos detectou uma proporção mais baixa do estágio I e uma proporção mais alta de estágios avançados de cânceres (estágio IIlb / IV) quando comparada à de intervalo anual, havendo diferença estatisticamente significante. Ademais, o período de 2,5 anos não mostrou significância estatística nas distribuições dos estágios em comparação com o de 2 anos.

Considerando que os intervalos mais longos são capazes de reduzir o efeito da triagem, o protocolo do BRELT1 também se propõe a analisar o impacto de períodos mais curtos entre os exames de TCBD, o que varia de 3 a 12 meses, conforme as características radiológicas e de risco para neoplasia de cada paciente (SANTOS et al., 2014, 2016; TANG et al., 2019).

\section{Critérios de Elegibilidade}

A eficácia da triagem na redução da mortalidade, demonstrada por evidências desses ECRs, fomentou organizações de saúde, como a American Cancer Society (ACS),a emitirem diretrizes de rastreamento do câncer de pulmão seguindo os requisitos de elegibilidade descritos no NLST: adultos com idade entre $55 \mathrm{e}$ 74 anos com boa saúde que atualmente fumam ou pararam nos últimos 15 anos; e têm pelo menos 30 anos de história de tabagismo; e recebem aconselhamento para cessação do tabagismo com base em evidências, caso sejam fumantes atuais; e foram submetidos a um processo de tomada de decisão informada/compartilhada que incluía informações sobre os possíveis benefícios, limitações e danos da triagem com o LDCT; e têm acesso a um centro de triagem e tratamento de alto volume e alta qualidade do câncer de pulmão (SMITH R, et al., 2019; BECKER N, et al., 2019).

Contudo, algumas diretrizes ainda divergem nos critérios clínicos, majoritariamente na idade em que se interrompe o rastreamento e no mínimo necessário de histórico de tabagismo frente à presença de fatores de risco adicionais para câncer de pulmão. Além disso, também há uma variação substancial em relação aos critérios radiológicos usados na avaliação dos exames de referência.

Alguns ECRs, como o BRELT1, avaliam variáveis como diâmetro do nódulo em mm, presença de espículas e localização em lobo superior; o que não são analisados em outros estudos. Dessa forma, a não padronização dos parâmetros clínicos e radiológicos configura-se como um empecilho na análise da real efetividade da triagem. Sendo necessário, portando, uma uniformização, a fim de mitigar essa heterogeneidade (SANTOS R, et al., 2014, SMITH R, et al., 2019; TANG X, et al., 2016).

\section{Desvantagens}

Ademais, devem-se analisar algumas desvantagens sobre o uso da TCBD como sua relação custobenefício, visto que a TC é considerada um exame de custo alto para a atenção básica. Além disso, há a possibilidade do surgimento de neoplasia devido à alta exposição à radiação ionizante, pois a dose de radiação produzida efetivamente pela TCBD em todo o organismo totaliza 1,5 mGy, em contraste com a produção pelo radiograma de tórax, que atinge valores de 0,02 mGy.

Sabe-se também que há alto risco de falsos positivos em nódulos acima de 4 a $6 \mathrm{~mm}$ e deve-se ponderar os danos que podem ser provocados a um paciente submetido a realização de biópsias pulmonares em doença benigna. $\mathrm{Na}$ atenção primária muitos médicos ainda não têm experiência para identificar os pacientes 
elegíveis para triagem ou não estão preparados para orientá-los no processo compartilhado de tomada de decisão sobre o rastreamento. Dessa forma, tais fatores devem ser considerados antes da implementação da triagem generalizada por TC de baixa dose (TANG X, et al., 2019).

O superdiagnóstico é também um fator que deve ser considerado, uma vez que em alguns casos pode ocorrer a detecção de lesões de baixa probabilidade de malignidade e de mortalidade erroneamente, situação onde o rastreamento propriamente dito não altera o prognóstico e a sobrevida do paciente de maneira significativa. De forma semelhante, pode ocorrer também a detecção de lesões indolentes ou de crescimento muito lento onde o aumento da sobrevida seria, em parte, atribuído a este crescimento indolente, não sendo, portanto, atribuído por completo ao diagnóstico e aos tratamentos precoces (JAMNIK S, et al., 2012).

\section{Vantagens}

Hoje existem evidências rigorosas que apoiam o valor da triagem para o câncer de pulmão com a TCBD, como a redução significante de $20 \%$ na mortalidade no estudo NLST e a redução moderada da mortalidade nos estudos europeus. Além disso, tal forma de rastreamento apresenta um benefício adicional, uma vez que proporciona um efeito positivo na cessação do tabagismo, devido ao resultado não intencional da triagem na mudança do estilo de vida e na redução ou abandono dos hábitos de fumar em populações elegíveis, que também gera uma redução nas mortes específicas por câncer de pulmão (BECKER N, et al., 2019; TANG X, et al., 2019; WENDER R, et al., 2013).

Diante dessas evidências de ECRs que mostram redução da mortalidade por câncer de pulmão e equilíbrio entre benefícios e danos potenciais, tem-se um cenário favorável para o uso da TCBD no rastreio desta neoplasia. Os Estados Unidos da América (EUA), fundamentados na diretriz da ACS 2013 com redação modificada em 2017, recomendam a triagem anual para câncer de pulmão com esse exame de imagem em populações selecionadas.

De forma similar, em 2017, a União Europeia declarou que o rastreamento do CP com TCBD começaria a ser implementado em toda a Europa o mais rápido possível. Todavia, no Brasil, ainda não existem numerosos estudos a respeito do rastreamento desse câncer, o que torna a conclusão do BRELT1 de fundamental importância para a obtenção de informações específicas que ratifiquem seu uso como ferramenta de rastreio de CP na população brasileira (SMITH RA, et al., 2019; TANG X, et al., 2019; SANTOS RS, et al., 2014, 2016).

\section{Distribuição da TC no país}

Apesar dos avanços na pesquisa sobre a efetividade da TCBD como ferramenta de rastreio do CP, é necessário compreender as limitações da realidade brasileira em relação ao acesso a esse exame de imagem. Dados mais recentes apontam que no ano de 2013 haviam 3217 tomógrafos em uso no país. Aproximadamente metade dos aparelhos estava disponível para uso pelos pacientes do SUS, contrastando com a parcela da população que depende do sistema público para ter acesso a cuidados de saúde (76\%) (DOVALES AC, et al., 2016; MARTINS LO, 2014).

Apesar de não haver orientações oficiais sobre esse indicador, o número de tomógrafos por milhão de pessoas no Brasil $(15,3)$ ainda se apresenta abaixo da média dos países membros da OECD (OECD Health at Glance, 2019). E esses números variam de acordo com a região do país. Enquanto no Sul, Sudeste e Centro Oeste encontram-se indicadores superiores a 18 TCs por milhão de pessoas, as regiões Norte e Nordeste não chegam a 10. Quando considerados apenas os tomógrafos a serviço do Sistema Único de Saúde (SUS), os valores são ainda menores, chegando a 6,1 no Norte e 5,6 no Nordeste, com uma média nacional de 9,3. (DOVALES AC, et al., 2016).

Esses números evidenciam a dificuldade de implantação no país de um programa de rastreio que dependa da TC. Outro aspecto que deve ser considerado é o custo-benefício do uso em larga escala do exame para rastreio na atenção primária.

Nos Estados Unidos, quando considerada população de 50-64 anos, estudo de 2012 julgou ser relevante o uso da TCBD como rotina de rastreio, quando comparado com o resultado obtido por outros programas de 
rastreio. Em estudo, de 2011, considerando população de 50-70 anos, apontaram que, se não acompanhado de cessação do tabagismo, o rastreamento com TCBD não atingiria números favoráveis, quando comparado com outros programas de rastreio (MCMAHON PM, et al., 2011; PYENSON BS, et al., 2012).

Na Nova Zelândia, estudo que incluiu população de 55-74 anos não se mostrou favorável para a implementação do programa de rastreio universal, evidenciando diferença da efetividade do método quando comparada a população nativa e não nativa da ilha (JAINE R, et al., 2020). No Brasil estudos nesse sentido são escassos, sendo necessária uma análise cuidadosa que leve em conta as particularidades de cada região do país.

\section{CONSIDERAÇÕES FINAIS}

Em conclusão, a literatura disponível é favorável ao uso de TCBD no rastreio do CP devido a sua sensibilidade superior a outros exames disponíveis, o que permite detecção e intervenção precoces. Entretanto, ainda são necessários novos estudos que se proponham a elucidar o impacto da exposição aumentada à radiação nos pacientes e a uniformizar os critérios radiológicos a serem utilizados. Ademais, é fundamental que literatura nacional traga informações específicas que ratifiquem o uso da TCBD como ferramenta local.

\section{REFERÊNCIAS}

1. ARAÚJO LH, et al. Câncer de pulmão no Brasil. Jornal Brasileiro de Pneumologia, 2018; 44:(1): 55-64.

2. BECKER N, et al. Lung cancer mortality reduction by LDCT screening - Results from the randomized German LUSI trial. International Journal of Cancer, 2020; 146(6): 1503-1513.

3. BRASIL. Ministério da Saúde. Instituto Nacional de Câncer José Alencar Gomes da Silva (INCA). Câncer de pulmão, 2020.

4. BRASIL. Ministério da Saúde. Instituto Nacional de Câncer José Alencar Gomes da Silva (INCA). Câncer de pulmão versão para Profissionais de Saúde, 2018.

5. BRASIL. Ministério da Saúde. Instituto Nacional de Câncer José Alencar Gomes da Silva (INCA). Tabagismo, 2020.

6. CENTERS FOR DISEASE CONTROL AND PREVENTION. Smoking \& Tobacco Use: Health Effects of Cigarette Smoking, 2017.

7. DOVALES AC, et al. Patterns and trends of computed tomography usage in outpatients of the Brazilian public healthcare system, 2001-2011. Journal of Radiological Protection, 2016; 36(3): 547-560.

8. DUMA N, et al. Non-Small Cell Lung Cancer: Epidemiology, Screening, Diagnosis, and Treatment. Mayo Clinic Proceedings, 2019; 94 (8): 1623-1640.

9. HASSAN LA, et al. Lung cancer: Biology and treatment options. Biochim Biophys Acta, 2015; 1856(2):189-210.

10. HONGLIN Z, et al. Comparison and Discussion of the Treatment Guidelines for Small Cell Lung Cancer. Thoracic Cancer, 2018; 9(7): 769-774

11. JAINE R, et al. Cost-effectiveness of a low-dose computed tomography screening programme for lung cancer in New Zealand. Lung Cancer Journal, 2018; 124: 233-240.

12. JAMNIK S, et al. Rastreamento Populacional e Diagnóstico Precoce no Câncer de Pulmão. Pneumologia Paulista, 2012; 26(1).

13. LIU Y, et al. Screening baseline characteristics of early lung cancer on low-dose computed tomography with computeraided detection in a Chinese population. Cancer Epidemiology, 2019; 62:1877-7821.

14. MARTINS LO. O Segmento da Medicina Diagnóstica no Brasil. Revista da Faculdade de Ciências Médicas de Sorocaba, 2014; 16(3):139-145.

15. MCMAHON PM, et al. Cost-effectiveness of computed tomography screening for lung cancer in the United States. Journal of Thoracic Oncology, 2011; 6: 1841-1848.

16. NASIM F, et al. Lung Cancer. Medical Clinics of North America, $2019 ; 103$ (3): 463-473.

17. NATIONAL CANCER INSTITUTE. Lung and Bronchus - Cancer Statistics Review, 2017.

18. NATIONAL HEALTH SERVICE. Lung Cancer, 2019.

19. OKEN MM, et al. Screening by chest radiograph and lung cancer mortality: The Prostate, Lung, Colorectal, and Ovarian (PLCO) randomized trial. Journal of the American Medical Association, 2011; 306(17):1865-73.

20. ORGANIZAÇÃO PAN-AMERICANA DE SAÚDE (OPAS). Folha Informativa - Câncer. OPAS/OMS Brasil, 2018.

21. ORGANIZATION FOR ECONOMIC COOPERATION AND DEVELOPMENT. OECD iLibrary, 2019. Health at a Glance.

22. ORTEGA-GÓMEZ A, et al. Gene-expression profiles in lung adenocarcinomas related to chronic wood smoke or tobacco exposure. Respiratory Research, 2016; 17:42.

23. PYENSON BS, et al. An Actuarial Analysis Shows That Offering Lung Cancer Screening As An Insurance Benefit Would Save Lives At Relatively Low Cost. Health affairs (Project Hope), 2012; 31(4): 770-9.

24. SANTOS RS, et al. Do Current Lung Cancer Screening Guidelines Apply for Populations With High Prevalence of Granulomatous Disease? Results From the First Brazilian Lung Cancer Screening Trial (BRELT1). The Annals of Thoracic Surgery, 2016; 101(2): 481-6. 
25. SANTOS RS, et al. Rastreamento de câncer de pulmão por meio de TC de baixa dosagem no Brasil: protocolo de pesquisa. Jornal Brasileiro de Pneumologia, 2014; 40(2): 196-199.

26. SILVA HAP, et al. O papel de marcadores tumorais no câncer de pulmão: revisão da literatura. Revista Médica de Minas Gerais, 2015; 25(4): 597-604.

27. SMITH RA, et al. Cancer screening in the United States, 2019: A review of current American Cancer Society guidelines and current issues in cancer screening. Cancer Journal for Clinicians publishes, 2019; 69(3):184-210.

28. TANG X, et al. Low-dose CT screening can reduce cancer mortality: A meta-analysis. Revista da Associação Médica Brasileira, 2019; 65(12): 1508-1514.

29. WENDER R, et al. American Cancer Society Lung Cancer: Screening Guidelines. Cancer Journal for Clinicians publishes, 2013; (63): 106-117.

30. WOODARD GA, et al. Lung Cancer Staging and Prognosis. Cancer Treatment and Research, 2016; (170): 47-75. 